Die Rolle der Wirtschaft in der Integrierten Produktpolitik

\section{Bottom-Up statt Top-down}

Produktbezogener Umweltschutz betrifft unmittelbar den Markt. Er berührt den Kern der Über-

\section{lebensfähigkeit der Unternehmen - die Leistungen für ihre Kunden. Integrierte Produktpolitik ist deshalb für den Bundesverband der Deutschen Industrie (BDI) ein ganz wichtiges Thema. Die Industrie hat die bisherige konzeptionelle Diskussion intensiv begleitet. Nun steht die Konkreti- sierung an.}

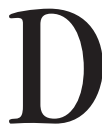

Von Thomas Becker

Erstens unterstützt der BDI den Grundgedanken einer integrierten Perspektive auf die Herstellung und Vermarktung zukunftsfähiger Produkte. Die Grenzen und die Defizite eines Vorgehens, dass nur an einzelnen Lebenszyklusphasen ansetzt, sind heute vor allem im Abfallbereich klar sichtbar. Solche Eingriffe können dazu führen, dass Entlastungen an einer Stelle mit Rückwirkungen an anderer Stelle erkauft werden, die aus einer integrierten Sicht den Sinn der Maßnahme in Frage stellen. Hinzu kommt, dass viele punktuell ansetzende Regelungen Investitionen in umweltorientierte Maßnahmen erzwingen, die einen wesentlich geringeren ökologischen „Return“ der verwendeten Mittel aufweisen als andere mögliche Maßnahmen. Schlüssel für den Erfolg ist die Integration umweltorientierter Analyse-Instrumente in den Produktentwicklungsprozess, um für jedes einzelne Produkt die jeweils optimalen Lösungen im Unternehmen zu ermitteln und zu realisieren.

Zweitens gilt: Produktpolitik ist Wirtschaftspolitik. Eine wesentliche Aufgabe für die deutsche Politik muss sein, die Wettbewerbsfähigkeit der deutschen Industrie zu stärken. Hierfür kann die im Vergleich zu ihren Wettbewerbern sehr hoch entwickelte Kompetenz der deutschen Unternehmen im Bereich des produktbezogenen Umweltschutzes als Basis genutzt werden. Die Industrie in Deutschland hat keinen Anlass, sich vor Anforderungen an eine nachhaltige Produktgestaltung zu verstecken. Es kommt aber darauf an, eine Politik so auszugestalten, dass diese Kompetenz auch im Wettbewerb zum Tragen kommt.

Ein warnendes Beispiel: Immer wieder wird als Vergleich für die Programmatik der Integration die Europäische IVU-Richtlinie zur integrierten Vermeidung und Verminderung von Umweltauswirkungen herangezogen. Diese behandelt die integrierte Betrachtung aller Umweltmedien im Bereich des Anlagengenehmigungsrechts. Gerade diese Richtlinie ist ein negatives Lehrstïck für eine reaktive Rolle der deutschen Politik gegenüber einer Konzeption aus dem angelsächsischen Raum. Sie erfordert - siehe die Diskussion um den Entwurf eines Umweltgesetzbuchs - erheblichen Anpassungsbedarf in Deutschland. Zugleich stärkt sie tendenziell die Wettbewerbsposition der Industrien anderer Länder. Aus diesem Fall ist beim Design einer integrierten Produktpolitik (IPP) zu lernen.

\section{Innovationsprozess als Basis}

Eine erfolgreiche Politik im Produktbereich kann drittens nicht Top-Down funktionieren. Vielmehr muss sie Bottom-Up auf dem Management der Umweltauswirkungen von Produkten und auf der Weiterentwicklung der hierfür bereitstehenden Instrumente in den Unternehmen setzen. Bei der Entwicklung einer erfolgreichen und zukunftsfähigen Politik muss daher zuerst gefragt werden, welche politisch definierten Faktoren eine eigenständige Identifizierung von ökologischen Problemen und Wegen zu ihrer Verringerung oder Beseitigung fördern bzw. behindern. Basis der Betrachtung muss also der Innovationsprozess im Unternehmen sein. Eine erfolgreiche IPP hat die Dynamik des Marktes zur Grundlage und ist darauf gerichtet, Innovationen schneller sowie ökologisch und ökonomisch effizienter zu erreichen. Ökologische Gesichtspunkte in einer wirtschaftlich Erfolg versprechenden Form zum Tragen zu bringen, ist entscheidend. Eine IPP, die anstrebt, unabhängig vom Marktgeschehen absolute Maßstäbe für das „Besser - Schlechter“ von Produkten zu definieren und durchzusetzen, wäre dagegen fehlgeleitet. Quoten sowie direkte und indirekte Stoffverbote sind für die Industrie kein Modellfall für eine zukunftsfähige Politik im produktbezogenen Umweltschutz.
Politik und Industrie müssen eine Integrierte Produktpolitik offensiv und konstruktiv angehen. Dafür gibt es neben der bereits angesprochenen integrierten Perspektive zwei weitere Schlüsselbegriffe: Kooperation und Kommunikation.

\section{Kooperation und Kommunikation}

Kooperation zwischen Politik und Wirtschaft ist der Schlüssel für eine erfolgreiche Politik im Produktbereich, denn: Es ist zu einfach, zwischen der Ebene der Politik, die Ziele definiert und den rechtlich-instrumentellen Rahmen schafft, auf der einen Seite und dem produktbezogenen Umweltmanagement der Unternehmen auf der anderen Seite zu unterscheiden. Diese Trennung verstellt leicht den Blick dafür, dass Innovationen von Unternehmen, die am Markt Erfolg haben, der entscheidende Engpassfaktor einer erfolgreichen Umweltpolitik im Produktbereich sind. Auf sie muss der politische Rahmen zugeschnitten werden, und zwar im Dialog von Politik und Wirtschaft.

Die zweite zentrale Aufgabe für Politik und Industrie ist die Verbesserung der Kommunikation entlang des Lebensweges und insbesondere zwischen Produzenten und Konsumenten. Die nachhaltige Optimierung von Produkten bedingt, die Fähigkeit der Unternehmen zu steigern, künftige ökologische Anforderungen an ihre Produkte frühzeitig wahrzunehmen und in ihren Entscheidungen zu berücksichtigen. Dabei ist dem BDI klar, dass Eigeninitiative und freiwillige Leistungen nur dann von den Bürgern auch als Alternative zu staatlicher Intervention anerkannt werden, wenn es gelingt, die Leistungen, die so erbracht wurden, auch in einer glaubwürdigen, transparenten und verständlichen Form zu kommunizieren. Ein Leitfaden zu den Spielregeln produktbezogener Umweltinformation, den Bundesumweltministerium und Umweltbundesamt sowie der BDI gegenwärtig erstellen, soll hierzu beitragen.

Die kommenden Monate werden Aufschluss darüber geben, ob und wie die drei Schlüsselfragen einer erfolgreichen IPP beantwortet werden. Der BDI wird sich hieran mit praktischen Beispielen aus Unternehmen und Verbänden beteiligen.

\section{Der Autor}

Dr. Thomas Becker ist Referent des Bundesverbands der Deutschen Industrie (BDI) in der Abteilung Umweltpolitik.

Kontakt: BDI, Abteilung Umweltpolitik, Breite Straße 29, 10178 Berlin, Tel. 030/ 2028-1481,

Fax -2481, E-mail: t.becker@bdi-online.de 
(c) 20I0 Authors; licensee IÖW and oekom verlag. This is an article distributed under the terms of the Creative Commons Attribution Non-Commercial No Derivates License (http://creativecommons.org/licenses/by-nc-nd/3.o/), which permits unrestricted use, distribution, and reproduction in any medium, provided the original work is properly cited. 